Draft Version June 17, 2020

Typeset using $\mathrm{LATEX}_{\mathrm{E}}$ default style in AASTeX63

\title{
On the Alignment of Galaxies in Clusters
}

\author{
Hrant M. Tovmassian $^{1}$ And J.P. Torres-Papaqui ${ }^{2}$ \\ 37r, W. California 30 \\ Glendale, CA, USA \\ ${ }^{2}$ Departamento de Astronomía, Universidad de Guanajuato \\ Apartado Postal 144, 36000, Guanajuato, Mexico
}

(Received; Revised; Accepted)

\begin{abstract}
We explore the distribution of position angles (PA) of galaxies in clusters. We selected for study the isolated clusters, since the distribution of the galaxy orientation in clusters with close neighbors could be altered by gravitational influence of the latter. We assume that galaxies are aligned, if their number at one $90^{\circ}$ position angle interval is more than twice higher than at the other $90^{\circ}$ interval. We study the galaxy PA distribution at the outer regions of clusters with smaller space density, where the probability of the PA variation in the result of interactions between galaxies is smaller than at the dense central regions. We found that the alignment of galaxies is more often observed in poor clusters and concluded that originally galaxies were aligned, but in the result of accretion in time of field galaxies with arbitrary orientations and also due to the mutual interactions the relative number of aligned galaxies decreases.
\end{abstract}

Keywords: galaxies: clusters - galaxies: alignment - galaxies: large-sale structure

\section{INTRODUCTION}

According to pancake scenario [1-3] galaxies form in the result of the gas-dust cloud collapse. At such case the angles (PA) of galaxies will naturally be aligned independent on the cluster mass. According to Miller \& Smith [4], Salvador-Sole \& Solanes [5] Usami \& Fujimoto [6], the galaxies could be aligned also at the hierarchical scenario of the cluster origin due to the cluster tidal field. At the latter scenario the galaxies will be aligned predominantly in rich clusters. Thus the alignment of galaxies in clusters is a clue for explanation of their origin. Therefore a lot of efforts have been undertaken in the past for study the distribution of the position angles (PA) of galaxies in clusters. Some evidence on the alignment of galaxies with the parent cluster were reported by Sastry [7] Adams, Strom \& Strom [8], Carter \& Metcalfe [9], Binggeli [10], Struble \& Peebles [11], Rhee \& Katgert [12], Lambas et al. [13], Flin \& Olowin [14], Fong, Stevenson \& Shanks [15]. More certainly the alignment was found between orientations of the cluster and of the BCG (cD) [7-9, 16-22]. Plionis et al.[23] and Rong, Zhang \& Liao [24] found an evidence that significant galaxy alignment is present in dynamically young clusters. Meanwhile, Dekel [25], van Kampen \& Rhee [26], Trevese, Cirimele \& Flin 27], Djorgovski [28] and Cabanela \& Aldering [29] found no galaxy alignment, except the alignment of the BCG with its parent cluster. Chen et al. [30] found a statistically significant galaxy-filament correlation, but not on the galaxy-cluster alignment. Thus, the results on the study of the galaxy alignment in clusters were contradictory.

In this paper we undertook new search for alignment of galaxies and showed that galaxies are aligned mostly in poor clusters. We suggest that the primordial orientations of member galaxies were ordered at the cluster origin, but later on the assembly of field galaxies by the cluster and interactions between galaxies within the cluster introduce disorder

Corresponding author: Hrant M. Tovmassian

htovmas@gmail.com 
in the galaxy orientations. We showed also that clusters in which the primordial alignment of galaxies preserved, do rotate.

\section{THE DATA}

We study the possible alignment of galaxies in ACO [31] clusters. Many ACO clusters are themselves clustered [32-34]. The gravitational influence of the nearby cluster may affect on the orientation of galaxies in the studied cluster. In order to avoid this effect we studied isolated clusters. We compiled a list of 73 strongly isolated clusters with nearest neighbor located on sky at the projected distance $>10 \mathrm{Mpc}$ (Table 1). For comparison we studied also the clusters with smaller degree of isolation, with 5 to $9 \mathrm{Mpc}$ projected distance to the nearest neighbor on sky. The list of the mild isolated 25 clusters is presented in Table ??. Redshifts of the selected clusters are $z<0.1$ and they contain more than 20 galaxies within area with $2 \mathrm{Mpc}$ radius. It is assumed that member galaxies of the most ACO clusters are located within $2 \mathrm{Mpc}$ of the Abell radius [32], defined as $R_{A}=1^{\prime} .7 / z\left(\mathrm{H}_{0}=72 \mathrm{~km} \mathrm{~s}^{-1} \mathrm{Mpc}^{-1}\right)$ [35]. The member galaxies of clusters were retrieved from the SDSS-DR9 [36]. The galaxies with the primary mode (marked in the catalog as 1) and good quality of observations (marked by 3) were retrieved. According to [37] we retrieved galaxies with velocities within $\pm 1500 \mathrm{~km} \mathrm{~s}^{-1}$ of the cluster velocity. PAs are those at $r$ band.

At the corresponding columns of Tables 1 and 2 the following information is given: the cluster designation, the redshift of the cluster and the number of galaxies in the cluster within $2 \mathrm{Mpc}$ radius. The parameters of clusters are from NED. ${ }^{1}$

\section{ANALYSIS}

We used a simple method for search of the alignment of the orientation of galaxies in clusters. We divided the range of PAs of galaxies in each cluster into two $90^{\circ}$ sections so that to have high number $\left(N_{h}\right)$ of galaxies at one section and small number $\left(N_{s}\right)$ of galaxies at the other section. We assume that there is an alignment signal, if the number of galaxies at one $90^{\circ}$ section is by at least 2 times higher than at the other $90^{\circ}$ section.

A primordial galaxy alignments in clusters could be severely damped by the violent relaxation, by the exchange of angular momentum in galaxy interactions over a Hubble time [38] that mostly occur in the dense cluster environment. Therefore, we first searched the orientation of galaxies in the outer area of clusters at the ring with cluster-centric radii $1 \div 2 \mathrm{Mpc}$.

The results of counts in the outer ring of clusters with smaller degree of isolation is presented in Table 4 . In the corresponding columns of Table 3 the following information is given: 1st - the cluster designation; $2 \mathrm{~d}$ - the interval of PAs at which the high number of galaxies are distributed; $3 \mathrm{~d}$ - the number $N_{h}$ of galaxies at this section; 4th the number of galaxies at the opposite section; 5th - the ratio $N_{h} / N_{s}$ at the searched region. In these clusters the alignment signal was found only for 8 out of 26 clusters, $32 \%$.

At the ring with cluster-centric radii $1 \div 2 \mathrm{Mpc} 43$ clusters out of 73 , i.e $59 \%$ have alignment signal (Table 3 ). Note that in the case of a random distribution of PAs the numbers of galaxies in two $90^{\circ}$ intervals could occasionally differ from each other by more than 2 times. In order to verify whether the the found number of clusters with alignment signal are real or are a result of random distribution of the galaxy PAs we applied non-parametric bootstrapping statistical test making 1000 simulations. The same statistical test was applies below for checking the reality of the found alignments in other cluster samples.

The probability that in 43 clusters out of 73 the ratio $N_{h} / N_{s}$ exceeds 2 is real and is not a result of a random distribution is $58.90 \%$ of success and a 95 percent confidence interval from $46.76 \%$ to $70.29 \%$ with a p-value $=0.01597$. The p-value or probability value $(¿ 0.05)$ is the probability of obtaining test results at least as extreme, as the results actually observed during the test, assuming that the null hypothesis is correct. Hence, the probability that the found galaxy alignments at rings are real, is sufficiently high.

The orientations of 19 clusters with alignment signal studied in this paper were determined by Plionis [19]. In Figure 1 the distribution of PAs of galaxies in these clusters and the PAs of the cluster large axis determined by Plionis [19] are shown. The PAs of the large axes of 14 clusters are within interval of PAs of aligned galaxies. For 2 clusters, A1783 and A1812, the PA of their large axes fall into the interval of PAs of aligned galaxies, if to take into account the errors of the PA measurements [19] about $30^{\circ}$. The PAs of large axes of only 3 clusters, A1126, A1139, and A1812 are out of the $90^{\circ}$ interval of the PAs of galaxies with alignment signal. The probability of 16 chance coincidences out

1 The NASA/IPAC Extragalactic Database (NED) is operated by the Jet Propulsion Laboratory, California Institute of Technology, under contract with the National Aeronautics and Space Administration. 
Table 1. The list of isolated clusters with nearby neighbor at projected distance $>10 \mathrm{Mpc}$.

\begin{tabular}{llllll}
\hline \hline Cluster & $z$ & $N_{2}$ & Cluster & $z$ & $N_{2}$ \\
\hline A595 & 0.0666 & 48 & A1552 & 0.0858 & 74 \\
A602 & 0.0619 & 62 & A1564 & 0.0792 & 52 \\
A634 & 0.0265 & 102 & A1599 & 0.0855 & 25 \\
A635 & 0.0925 & 34 & A1609 & 0.0891 & 27 \\
A660 & 0.0642 & 25 & A1616 & 0.0833 & 48 \\
A671 & 0.0502 & 98 & A1630 & 0.0648 & 36 \\
A690 & 0.0788 & 49 & A1684 & 0.0862 & 27 \\
A692 & 0.0894 & 50 & A1692 & 0.0842 & 49 \\
A695 & 0.0687 & 27 & A1750 & 0.0852 & 91 \\
A699 & 0.0851 & 31 & A1781 & 0.0618 & 45 \\
A724 & 0.0933 & 46 & A1783 & 0.0690 & 50 \\
A727 & 0.0951 & 58 & A1809 & 0.0791 & 94 \\
A744 & 0.0729 & 32 & A1812 & 0.0630 & 28 \\
A757 & 0.0517 & 49 & A1825 & 0.0595 & 30 \\
A779 & 0.0225 & 115 & A1827 & 0.0654 & 41 \\
A819 & 0.0759 & 20 & A1849 & 0.0963 & 27 \\
A834 & 0.0709 & 35 & A1864 & 0.0870 & 51 \\
A858 & 0.0863 & 26 & A1890 & 0.0574 & 83 \\
A1024 & 0.0734 & 49 & A2018 & 0.0878 & 50 \\
A1028 & 0.0908 & 26 & A2019 & 0.0807 & 24 \\
A1035 & 0.0684 & 59 & A2022 & 0.0578 & 78 \\
A1066 & 0.0690 & 83 & A2048 & 0.0972 & 61 \\
A1126 & 0.0646 & 33 & A2082 & 0.0862 & 24 \\
A1139 & 0.0398 & 50 & A2107 & 0.0411 & 130 \\
A1142 & 0.0349 & 64 & A2108 & 0.0919 & 48 \\
A1168 & 0.0906 & 41 & A2110 & 0.0980 & 27 \\
A1169 & 0.0586 & 79 & A2122 & 0.0661 & 72 \\
A1238 & 0.0733 & 68 & A2142 & 0.0909 & 123 \\
A1270 & 0.0692 & 63 & A2148 & 0.0877 & 30 \\
A1307 & 0.0817 & 67 & A2162 & 0.0322 & 47 \\
A1314 & 0.0335 & 119 & A2178 & 0.0928 & 24 \\
A1371 & 0.0398 & 61 & A2205 & 0.0876 & 39 \\
\hline \hline & 0.0768 & 72 & A2255 & 0.0806 & 122 \\
A1480 & 0.0734 & 31 & A2366 & 0.0529 & 53 \\
A1507 & 0.0604 & 57 & A2593 & 0.0413 & 138 \\
A 15769 & 60 & A2630 & 0.0667 & 37 \\
& & & & & \\
\hline
\end{tabular}

of 19 is sufficiently small, 0.01. Even with exclusion of A1783 and A1812, the probability of the chance coincidences of 14 out of 19 is still small, 0.02. The coincidence of the cluster large axes orientation with the interval of PAs of the majority of the cluster galaxies shows that the applied simple method for searching the alignment of galaxies in clusters is reliable.

The results of counts in the outer ring of clusters with smaller degree of isolation and at the central area of strongly isolated clusters are presented respectively in Table 4 and Table 5 identical to Table 1.

In the outer ring of clusters with smaller degree of isolation the alignment signal is found only for 8 out of 25 clusters, $32 \%$. The probability that the found alignment signal in the ring of 8 out of 25 clusters is real, is $32.00 \%$ of success with a 95 percent confidence interval from $14.94 \%$ to $53.50 \%$ with a p-value $=0.01078$. 


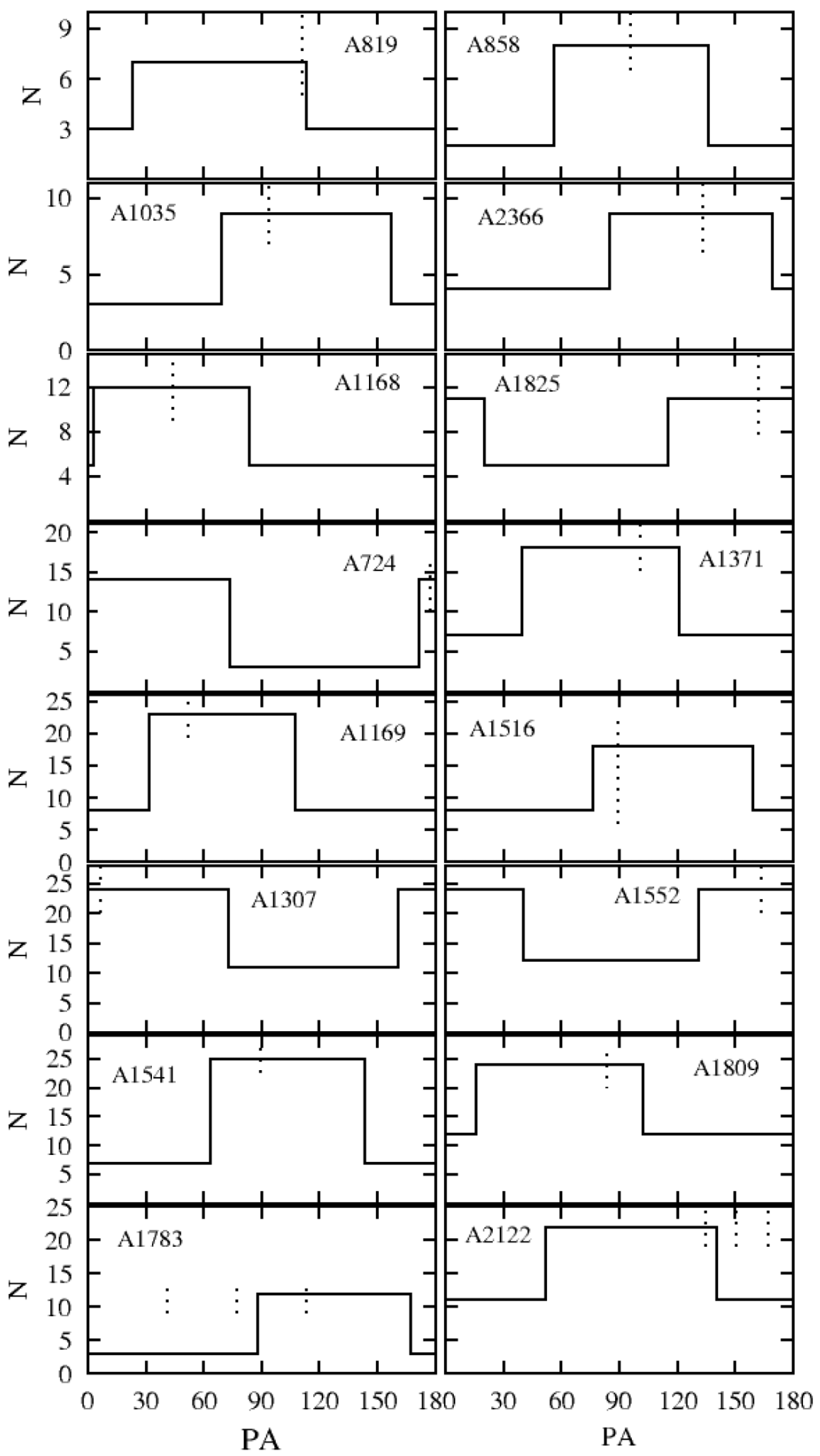

Figure 1. The comparison of the distribution of PAs of galaxies in clusters with alignment signal with the cluster PA from Plionis [19] shown by dotted line. In cases of A1783 and A2122 the interval of the errors of the PA of the large axes of the cluster are also shown. 
Table 2. The list of mild isolated clusters with nearby neighbor at projected distance $5<d<9$ Mpc.

\begin{tabular}{llllll}
\hline \hline Cluster & $z$ & $N_{2}$ & Cluster & $z$ & $N_{2}$ \\
\hline \hline A912 & 0.0446 & 21 & $\mathrm{~A} 1691$ & 0.0721 & 75 \\
$\mathrm{~A} 933$ & 0.0956 & 47 & $\mathrm{~A} 1775$ & 0.0717 & 60 \\
$\mathrm{~A} 1100$ & 0.0463 & 53 & $\mathrm{~A} 1795$ & 0.0625 & 103 \\
$\mathrm{~A} 1149$ & 0.0710 & 37 & $\mathrm{~A} 1831$ & 0.0615 & 37 \\
$\mathrm{~A} 1185$ & 0.0325 & 182 & $\mathrm{~A} 1927$ & 0.0945 & 35 \\
$\mathrm{~A} 1205$ & 0.0754 & 46 & $\mathrm{~A} 1983$ & 0.0436 & 149 \\
$\mathrm{~A} 1267$ & 0.0329 & 28 & $\mathrm{~A} 1991$ & 0.0587 & 99 \\
$\mathrm{~A} 1291$ & 0.0527 & 84 & $\mathrm{~A} 2029$ & 0.0773 & 77 \\
$\mathrm{~A} 1468$ & 0.0844 & 31 & $\mathrm{~A} 2065$ & 0.0726 & 115 \\
$\mathrm{~A} 1589$ & 0.0725 & 100 & $\mathrm{~A} 2089$ & 0.0711 & 60 \\
$\mathrm{~A} 1638$ & 0.0620 & 33 & $\mathrm{~A} 2092$ & 0.0669 & 50 \\
$\mathrm{~A} 1650$ & 0.0838 & 59 & $\mathrm{~A} 2149$ & 0.0679 & 40 \\
$\mathrm{~A} 1663$ & 0.0843 & 60 & & & \\
\hline \hline
\end{tabular}

At the central region of strongly isolated clusters the alignment signal is found for 27 clusters, $36 \%$, out of the studied 73. The probability that the alignment at the central area of clusters does not occur by chance and is real is $36.98 \%$ of success and a 95 percent confidence interval from $25.97 \%$ to $49.08 \%$ with a p-value $=0.01442$. Hence, the found alignment of galaxies in both cluster samples are caused rather by random distribution of the galaxy PAs and are not real.

\subsection{The dependence of the alignment on the cluster richness}

The alignment of galaxies could depend on the richness of clusters and/or on the absolute magnitude of the observed galaxies, i.e. on the cluster redshift. In order to find out whether the alignment signal depends on the cluster richness or distance we split the list of 73 strongly isolated clusters into two parts: with low and high redshift clusters, and also poor and rich ones. In the consecutive lanes of Table 6 the average redshift $z$, the average number $N_{2}$ of galaxies, the minimal absolute magnitude $\left\langle M_{r}\right\rangle$ in $r$-band for the average redshift, the number $N_{a s}$ of galaxies with alignment signal and the ratio $N_{a s} / N_{t}$ of the number of clusters with alignment signal to the total number of clusters are presented for nearby (column 2) and distant (column 3) clusters. In Table 7 the same data are presented for samples of rich and poor clusters.

Table 6 shows that difference between relative numbers of galaxies with alignment signal in nearby and distant clusters is not high, although the distant clusters are on average by 1.5 times farther and the limiting absolute $M_{r}$ magnitudes of galaxies in this clusters differ by about $1^{m}$. The average total numbers of galaxies in clusters of both samples also does not differ from each other significantly. The relative number of nearby clusters with alignment signal is 0.51 with $50.06 \%$ of success and 95 percent confidence interval from $38.71 \%$ to $62.59 \%$ with a p-value $=$ 3.182e-03. The relative number of distant clusters with alignment signal is 0.65 , with $64.38 \%$ of success and a 95 percent confidence interval from $52.30 \%$ to $75.25 \%$ with a p-value $=1.818 \mathrm{e}-04$.

The situation is different when we compare rich and poor clusters. Table 7 shows that the differences between the average redshifts and the limiting absolute magnitudes $M_{r}$ of these two samples are smaller in comparison to those in nearby and distant clusters. However, the relative number of poor clusters with alignment signal is by about 2.6 times higher in comparison to rich ones. The relative number of rich clusters with alignment signal is 0.40 with $39.72 \%$ of success and a 95 percent confidence interval from $28.45 \%$ to $51.85 \%$ with a p-value $=1.173 \mathrm{e}-02$. The relative number of poor clusters with alignment signal is 0.76 with $75.34 \%$ of success and a 95 percent confidence interval from $63.85 \%$ to $84.68 \%$ with a p-value $=1.514 \mathrm{e}-085$. Thus, in poor clusters the probability of the reality of the found alignment is sufficiently high, about $80 \%$.

\section{DISCUSSION AND CONCLUSIONS}

By study of the distribution of PAs of galaxies in the ring with radii $1 \div 2 \mathrm{Mps}$ of 73 strongly isolated clusters the alignment signal is found in 43 clusters, i.e. in about $60 \%$. Such high number of clusters with aligned galaxies may not be caused by a chance distribution of the galaxy PAs. Among the less isolated clusters and in the central dense area of 
Table 3. The list of strongly isolated clusters with alignment signal for galaxies at the ring with radii $1 \div 2 \mathrm{Mpc}$.

\begin{tabular}{|c|c|c|c|c|}
\hline Cluster & $I_{P A}$ & $N_{h}$ & $N_{s}$ & $\left(\frac{N_{h}}{N_{s}}\right)_{1 \div 2}$ \\
\hline & degree & & & \\
\hline A595 & $37-122$ & 12 & 3 & 4.0 \\
\hline A635A & $78-165$ & 8 & 2 & 4.0 \\
\hline A660A & $160-69$ & 12 & 4 & 2.2 \\
\hline A695A & 26-107 & 9 & 3 & 3.0 \\
\hline A699A & $82-158$ & 12 & 6 & 2.0 \\
\hline A724 & $171-81$ & 14 & 3 & 4.7 \\
\hline $\mathrm{A} 744 \mathrm{~A}$ & $2-86$ & 7 & 3 & 2.3 \\
\hline A819A & 23-113 & 7 & 3 & 2.3 \\
\hline A834 & $18-106$ & 15 & 4 & 3.75 \\
\hline A858 & $56-136$ & 8 & 2 & 4.0 \\
\hline A1028A & $1-89$ & 9 & 4 & 2.25 \\
\hline A1035A & $69-158$ & 8 & 4 & 2.0 \\
\hline A1126A & $18-108$ & 11 & 5 & 2.2 \\
\hline A1139 & $6-96$ & 12 & 6 & 2.0 \\
\hline A1168 & $3-84$ & 12 & 5 & 2.4 \\
\hline A1169 & 20-108 & 23 & 8 & 2.9 \\
\hline A1238A & $102-10$ & 23 & 7 & 3.3 \\
\hline A1270 & 69-159 & 17 & 7 & 2.4 \\
\hline A1307 & $164-74$ & 24 & 11 & 2.2 \\
\hline A1371 & $39-121$ & 18 & 7 & 2.6 \\
\hline A1480A & $161-70$ & 11 & 5 & 2.2 \\
\hline A1516A & $76-160$ & 18 & 8 & 2.25 \\
\hline A1541 & $64-144$ & 25 & 7 & 3.6 \\
\hline A1552 & $131-41$ & 24 & 12 & 2.0 \\
\hline A1599A & $100-3$ & 7 & 3 & 2.3 \\
\hline A1616A & $2-89$ & 16 & 6 & 2.7 \\
\hline A1684A & $68-156$ & 8 & 4 & 2.0 \\
\hline A1781A & $68-156$ & 19 & 7 & 2.7 \\
\hline A1783A & 88-167 & 12 & 3 & 4.0 \\
\hline A1809A & $15-103$ & 24 & 12 & 2.0 \\
\hline A1812A & $32-122$ & 12 & 4 & 3.0 \\
\hline A1825A & $115-20$ & 11 & 5 & 2.2 \\
\hline A1849A & $73-150$ & 9 & 2 & 4.5 \\
\hline A2018A & $44-131$ & 21 & 7 & 2.6 \\
\hline A2019A & $75-156$ & 9 & 4 & 2.25 \\
\hline A $2082 \mathrm{~A}$ & $144-40$ & 11 & 1 & 11.0 \\
\hline A2108 & $32-108$ & 12 & 3 & 4 \\
\hline A2110 & $91-180$ & 11 & 3 & 3.7 \\
\hline $\mathrm{A} 2122 \mathrm{~A}$ & 51-141 & 22 & 11 & 2.0 \\
\hline A 2148 & $108-18$ & 8 & 3 & 2.3 \\
\hline $\mathrm{A} 2178 \mathrm{~A}$ & 51-133 & 9 & 4 & 2.25 \\
\hline $\mathrm{A} 2366 \mathrm{~A}$ & $85-170$ & 9 & 4 & 2.25 \\
\hline A2630A & 44-132 & 10 & 2 & 5 \\
\hline
\end{tabular}


Table 4. The list of the mild isolated clusters with alignment signal for galaxies at the ring with radii $1 \div 2 \mathrm{Mpc}$.

\begin{tabular}{lllll}
\hline \hline Cluster & $I_{P A}$ & $N_{h}$ & $N_{s}$ & $\left(\frac{N_{h}}{N_{s}}\right)_{1 \div 2}$ \\
\hline \multicolumn{5}{c}{ degree } \\
\hline \hline A933 & $24-111$ & 18 & 8 & 2.25 \\
A1100 & $83-180$ & 15 & 7 & 2.1 \\
A1119 & $63-157$ & 8 & 4 & 2.0 \\
A1205 & $96-171$ & 8 & 4 & 2.0 \\
A1267 & $43-140$ & 13 & 4 & 3.25 \\
A1468 & $124-6$ & 9 & 4 & 2.25 \\
A1775 & $45-132$ & 37 & 17 & 2.2 \\
A1927 & $57-141$ & 17 & 4 & 4.25 \\
A2149 & $16-104$ & 12 & 4 & 3.0 \\
\hline \hline
\end{tabular}

Table 5. The list of clusters with aligned galaxies at the cluster central region.

\begin{tabular}{lllll}
\hline \hline Cluster & $I_{P A}$ & $N_{h}$ & $N_{s}$ & $\left(\frac{N_{h}}{N_{s}}\right)_{1}$ \\
& degree & & & \\
\hline \hline A595 & $37-122$ & 12 & 3 & 2.1 \\
A660A & $160-69$ & 12 & 4 & 2.0 \\
A695A & $26-107$ & 9 & 3 & 2.7 \\
A727 & $16-106$ & 22 & 17 & 2.8 \\
A744A & $2-86$ & 7 & 3 & 4.5 \\
A834 & $18-106$ & 15 & 4 & 3.0 \\
A1028A & $1-89$ & 9 & 4 & 2.25 \\
A1126A & $18-108$ & 11 & 5 & 3.0 \\
A1168 & $3-84$ & 12 & 5 & 2.7 \\
A1238A & $102-10$ & 23 & 7 & 2.75 \\
A1371 & $39-121$ & 18 & 7 & 2.0 \\
A1541 & $64-144$ & 25 & 7 & 2.5 \\
A1552 & $131-41$ & 24 & 12 & 2.2 \\
A1564A & $32-122$ & 13 & 12 & 2.25 \\
A1599A & $100-3$ & 7 & 3 & 2.0 \\
A1616A & $2-89$ & 16 & 6 & 2.7 \\
A1630A & $7-96$ & 7 & 4 & 2.6 \\
A1692 & $9-96$ & 17 & 9 & 3.0 \\
A1750A & $13-103$ & 36 & 23 & 2.7 \\
A1781A & $68-156$ & 19 & 7 & 2.1 \\
A1812A & $32-122$ & 12 & 4 & 3.0 \\
A2019A & $75-156$ & 9 & 4 & 2.7 \\
A2048 & $0-90$ & 19 & 14 & 2.1 \\
A2082A & $144-40$ & 11 & 1 & 3.0 \\
A2108 & $32-108$ & 12 & 3 & 3.1 \\
A2122A & $51-141$ & 22 & 11 & 2.2 \\
A2205A & $46-136$ & 14 & 10 & 2.0 \\
\hline \hline
\end{tabular}


Table 6. The parameters of the nearby and distant clusters.

\begin{tabular}{rrr}
\hline \hline & Nearby clusters & Distant clusters \\
\hline$\langle z\rangle$ & $0.0565 \pm 0.0143$ & $0.0860 \pm 0.0066$ \\
$\left\langle N_{2}\right\rangle$ & $62 \pm 30$ & $50 \pm 26$ \\
$\left\langle M_{r}\right\rangle$ & -19.09 & -20.02 \\
$N_{\text {as }}$ & 19 & 24 \\
$N_{\text {as }} / N_{t}$ & $0.51 \pm 0.12$ & $0.65 \pm 0.13$ \\
\hline \hline
\end{tabular}

Table 7. The parameters of poor and rich clusters.

\begin{tabular}{rrr}
\hline \hline$z$ & Rich clusters & Poor clusters \\
\hline$\left\langle N_{2}\right\rangle$ & $77 \pm 25$ & $35 \pm 9$ \\
$\left\langle M_{r}\right\rangle$ & -19.47 & -19.78 \\
$N_{a s}$ & 15 & 28 \\
$N_{a s} / N_{t}$ & $0.40 \pm 0.10$ & $0.76 \pm 0.14$ \\
\hline \hline
\end{tabular}

clusters with $1 \mathrm{Mpc}$ radius the alignment signal is found respectively in about $37 \%$ and $29 \%$ of clusters, that is close to the expected number of a chance occurrence of the alignment signal, the ratio $N_{h} / N_{s}>2$. The separate analysis of clusters of different richnesses and distances showed that the alignment depends on the cluster richness. Alignment is found in about $75 \%$ of poor clusters with on average 35 galaxies within $2 \mathrm{Mpc}$. The probability that this is not due by random distribution of the galaxy PAs is suficiently high. This evidences in favor of the pancake scenario [1,2] of the cluster formation. If so, clusters could preserve the angular momentum of the primordial gas cloud.

According to Miller \& Smith [4], Salvador-Sole \& Solanes [5] Usami \& Fujimoto [6], the galaxies could as well be aligned in the hierarchical scenario due to the tidal field of the cluster. However, the tidal field of the cluster would apparently be more effective in rich clusters with higher mass and the alignment would be observed in rich clusters. Whereas, we found the opposite.

During the cluster evolution the primordial alignment of galaxies could be altered. The alignment rate will decrease in the result of gravitational influence of nearby clusters and mutual interactions between galaxies. Apparently the rate of interactions is higher in rich clusters and especially at the cluster dense central regions. The gravitational influence would apparently have smaller effect on the orientation of massive galaxies. Therefore, the alignment of only very massive BCGs (cDs) has been found with the cluster orientation [7-10, 17-22]. The inclusion to the cluster content of the faint field galaxies by the hierarchical assembly [39 and references therein] with arbitrary orientations will certainly decrease the relative number of aligned galaxies. The poorer is the cluster, i.e. the less massive it is, the smaller amount of field galaxies would be assembled. Thus, the primordial alignment is better preserved in poor clusters, in which both reasons for altering it, interactions between galaxies and assembly of the field galaxies are less effective.

\section{ACKNOWLEDGMENTS}

We are grateful to M. Plionis for presentation the list of clusters with their neighbors and to the anonymous referee for careful reading of the manuscript and valuable comments. T-P acknowledges for support through grant DAIPUGto (0173/19). This research has made use of the NASA/IPAC Extragalactic Database (NED), which is operated by the Jet Propulsion Laboratory, California Institute of Technology, under contract with the National Aeronautics and Space Administration. Funding for SDSS-III has been provided by the Alfred P. Sloan Foundation, the Participating Institutions, the National Science Foundation, and the U.S. Department of Energy Office of Science. The SDSS-III web site is http://www.sdss3.org/.

SDSS is managed by the Astrophysical Research Consortium for the Participating Institutions of the SDSS- III Collaboration including the University of Arizona, the Brazilian Participation Group, Brookhaven National Laboratory, Carnegie Mellon University, University of Florida, the French Participation Group, the German Participation Group, 
Harvard University, the Instituto de Astrofisica de Canarias, the Michigan State/Notre Dame/JINA Participation Group, JohnsHopkins Univirsity, Lawrence Berkeley National Laboratory, Max Planck Institute for Astrophysics, Max Planck Institute for Extraterrestrial Physics, New Mexico State University, New York University, Ohio State University, Pennsylvania State University, University of Portsmouth, Princeton University, the Spanish Participation Group, University of Tokyo, University of Utah, Vanderbilt University, University of Virginia, University of Washington, and Yale University.

\section{REFERENCES}

1. Zel'dovich, Ya. B., 1970, A\&A, 5, 84 2. Zel'dovich, Ya. B., Einasto, J., \& Shandarin, S. F. 1982, Nature, 300, 407 3. Doroshkevich, A.G., Shandarin, S, \& Saar, E. 1978, MNRAS, 184, 64 4. Miller, R. H., \& Smith, B. H. 1982, ApJ, 253, 58 5. Salvador-Sole, E. \& Solanes, J.M., 1993, ApJ, 417, 427 6. Usami, M. \& Fujimoto, M., 1997, ApJ, 487, 489 7. Sastry, G. N. 1968, PASP, 80, 252 8. Adams, M.T., Strom, K.M., \& Strom, S.E., 1980, ApJ, 238, 445 9. Carter, D., \& Metcalfe, J. 1980, MNRAS, 191, 32510. Binggeli, B. 1982, A\&A, 107, 338 11. Rhee, G. F. R. N., \& Kartger, P. 1987, A\&A, 183, 217 12. Lambas, D. G., Groth, E. J., \& Peebles, P. J. E. 1988, AJ, 95, 99613. Struble, M. F., \& Peebles, P. J. E., 1985, AJ, 90, 58214. Flin, P., \& Olowin, R. P., 1991, in Physical Cosmology, edited by A. Blanchard, L. Celniker, M. Lachieze-Roy, and J. Tran Van Lan (Editions Frontieres, Gifsur-Yvette), p. 512 15. Fong, R., Stevenson, P. R. F., \& Shanks, T. 1990, MNRAS, 242, 146 16. Struble, M.F., 1990, AJ, 99, 743 17. West, M. J., 1989, ApJ, 347, 610 18. West, M. J., 1994, MNRAS, 268, 79 19. Plionis M., 1994, ApJS., 95, 401 20. Fuller, T.M., West, M.J. \& Bridges, T.J., 1999, ApJ, 519, 22 21. Kim, R.S.J., et al. (SDSS collaboration), 2001, BAAS, 33, 152122. Chambers, S.W., Melott, A.L., Miller, C.J., 2002, ApJ, 565, 849 23. Plionis M., Benoist C., Maurogordato S., Ferrari C., \& Basilakos S. 2003, ApJ, 594, 144. 24. Rong, Y., Zhang, S.-N., \& Liao, J.-Y., 2015, arXiv:1507.07418 25. Dekel, A., 1985, ApJ, 298, 461 26. van Kampen, E., Rhee, G.F.R.N., 1990, A\&A, 237, 283 27. Trevese, D., Cirimele, G., \& Flin, P., 1992, AJ, 104, 93528.

Djorgovski, S.G., 1987, NNGP Proc. 227 29. Cabanela, J. E. \& Aldering, G., 1998, AJ, 116, 1094 30. Chen, Y.C., et al. 2019, MNRAS, 485, 2492 31. Abell G. O., Corwin H. G., Jr., \& Olowin R. P. 1989, ApJS, 70, 138 32. Abell, G. O., 1958, ApJS, 3, 211 33. Bogart, R. S., \& Wagoner, R. V., 1973, Ap. J., 181, 609 34. Hauser, M. G., \& Peebles, P. J. E., 1973, Ap. J., 185, 757 35. Andernach, H., Waldhausen, H. \& Wielebinski, R.,1980, A\&AS, 41, 339 36. Ahn et al. 2012, ApJS, 203, 21 37. Collins, C. A., et al., 1995, MNRAS, 274, 107 38.Coutts, A., 1996, MNRAS, 278, 87 39. Kravtsov, A.V., \& Borgani, S., 2012, ARA\&A, 50, 353 